\title{
Study on the Influence and Promotion of Chinese Traditional Martial Arts in Different Groups
}

\author{
Zijian Chang ${ }^{1, a}$ and Yan $\mathrm{Li}^{2, \mathrm{~b}}$ \\ ${ }^{1}$ School of Northwest Minzu University, Gansu 731000, China; \\ ${ }^{2}$ School of Shanxi Xueqian Normal University, Shanxi 710100, China; \\ a450142475@qq.com, ${ }^{b} 522269873 @ q q . c o m$
}

\section{Keywords: Martial arts; Different people; Influence; Promotion}

\begin{abstract}
Based on the Chinese traditional martial arts, this paper makes an analysis of the formation of the people's fitness habit and finds out the good habits of Chinese traditional martial arts. Mainly uses the literature material method and logic analysis of martial arts fitness habits construction are studied and discussed, combined with the characteristics in the new period, to build a lifelong habit of Chinese traditional martial arts movements, in order to achieve the Chinese traditional martial arts sports to human body health of body and mind harmonious development purposes.
\end{abstract}

\section{Introduction}

Be health problems in the world people must focus on the topic, in order to clear understanding of the relation between Chinese traditional martial arts exercise habits and health and health problems when should take reasonable solution to the Chinese traditional martial arts exercise habits to promote physical and mental health and put forward corresponding to advice.

\section{The Historical Source of the Movement of Chinese Traditional Martial Arts}

The origin of martial arts can be traced back to prehistoric times. It was the production activities of primitive society and primitive military war that promoted the germination of martial arts. In the Xia, Shang and Zhou Dynasties, the form of martial arts has been gradually delineated by history, and frequent wars have become increasingly dependent on it. In education, Zhou Dynasty set up special departments, such as "new schools", "new schools" and "order", etc., and put the shooting, royal and traditional dance of martial arts in the column of education, emphasizing that physical fitness is the foundation of the activity.

"Heaven is healthy, the gentleman to strive for self-improvement" is to say that the day is vigorous, the movement is not only, the human also should show the active state of activity. During the warring states period, wars often make the ruling class had to soldier physical quality and combat skills put forward higher request, be incorporated in the military martial arts training, although accompanied by imposing the will of the ruling class, but on the other hand, shows the official ruling class for soldiers group attaches great importance to life. The rulers of the Qin Dynasty sought to create a fertile soil for the spread of sword culture throughout the country by promulgating decrees and decrees, which played a positive role in cultivating people's practice of swordplay.

Qin Dynasty when the arrival of the activity for Angle to launch "primitive Angle offset, is not only for ancestor worship of totem of emotions, and is as a display of their own exuberant vitality and vent". At the end of The Eastern Han Dynasty, Hua tuo created the five animals with high fitness value. Of enforcement, in ninety, fresh and bright, teeth after established "the health point of view has been widely implemented at that time, many people lifelong Kungfu preserve one's health, keeping in good health method for people health effects are obvious. The martial arts of The Three Kingdoms period already from the pre-Qin period on the "cut neck, easy liver lung" turned into "fold a bamboo sword", "rod for sugarcane" gentleman's play, this is a brutal fight towards 
civilization sport evolution, show the human care of own life at that time.

Northern and Southern Dynasties, minority and Han nationality fusion, martial arts further development, the selection of literary works in the word "martial arts", martial arts has been forming technology and the theory of general architecture, martial arts is the fitness function. "Continued monk biography" in "the house standing, the monk zhai, king and his wife, hand to feed, digestion after a meal, learning the martial arts" at palace dignitaries have through practicing Wu Shulai guarantee health fitness habits. Tang mu zong was in yuan and 15 February, "the lucky left god army, the Angle of view and the mixed drama, the day of the day" showed the entertainment and fitness function of the Angle of the play was recognized by the ruler. Angle for activities in the folk widely certainly "hungry ghost festival in July, the common good" sumo wrestling, YunQiu miasma "also, the ancients think sumo wrestling the fitness activities can effectively resist the autumn season" malaria ", Angle for activities has become a particular holiday people pursue health and held a national custom.

The Ming Dynasty is a stage of rapid development of Chinese martial arts. Among them, "Wang Zhengnan epitaph" first proposed the theory of internal boxing, which recorded that there were many martial artists who had practiced their inner family Kungfu to prolong their life and strengthen their body. The shaolin temple, as a universal religious organization, has been used by monks to strengthen the body and protect the home. In the Qin Dynasty, military sports began to strengthen their functions, and folk martial arts began to be separated from martial arts, and integrated into religious culture, philosophy and other aspects. Due to the influence of folk religious culture and the acceptance of the traditional cultural thought, Chinese traditional martial arts began to focus on promoting "qi" and focusing on health preservation. With the development of firearms, the status of cold weapon gradually weakened, and people became more and more aware of the importance of fitness value in martial arts.

\section{Modern Inheritance of the Fitness Philosophy of Martial Art}

During the period of the republic of China, brush the new Chinese martial arts, combines the traditional martial arts and soldier type gymnastics, created a new beginning of martial arts, although later do not tally with the concept of the new culture movement and the tathagata collapse resistance, but its about combination of Chinese and western concept of martial arts and the modern sports fitness idea coincides with mine. In 1912, Nanyang public school (the predecessor of Shanghai Jiaotong university) formally established the technical department and insisted on the tenet of "carrying forward the national essence and strengthening the body". After the establishment of Huo yuanjia Chin Woo Athletic association, "to promote the martial arts, research the sports, and forge a strong national essence". Then, in 1924, he put forward the slogan "the science of the state is scientific and the one million people", and the Chinese traditional martial arts movement was gradually referred to the strategic height of the strong people. In 1949, the People's Republic of China was founded, and martial arts was highly valued by party and government organs. Traditional martial arts of management by the national martial arts association also sent to the provinces martial arts association management, the administration of the provinces and cities have overlapping communication, transmission, in improving people's health at the same time, greatly enriched the people's cultural life, to a certain period the spirit of building has more positive impact.

\section{The Promotion of Chinese Traditional Martial Arts.}

Nowadays, the pressure of social competition is huge, and people are running on the highway of work and study, and the attention to their own health problems is reduced. The crowd of all ages have appeared because of the lack of exercise for a long time of work, study, through the Chinese traditional martial arts movement can alleviate or even improve our own health problems and hidden dangers. The identity of the martial arts to adapt to the range to a person's age is divided into children, young and middle-aged and elderly, according to different age stages with different Chinese traditional martial arts sports effectively promote the health level. 
The Influence of Chinese Traditional Martial Arts Programs on Teenagers in School Sports . School sports is the foundation of lifelong sports. In 1956, the ministry of education compiled and promulgated the syllabus for the physical education of middle and primary schools, and first mentioned the content requirements of martial arts. In 1961, martial arts were included in the national curriculum for physical education, which was published in the same year. In the outline, martial arts teaching also has a clear stipulation of the necessary hours. In the teaching content, there are certain learning requirements for the basic skills, basic movements, combination movements, martial arts and primary boxing.

The development of cardiopulmonary function in the adolescent stage is not complete, and the quality of endurance, speed, explosive force and strength can greatly improve the space, and the development of physical quality is not comprehensive. Martial arts in the long fist project can be a good exercise the student's physical quality, in the process of practicing long fist requires fast, The hand should be quick, the eyes should be sharp, the body should be flexible, the step should be stable, the force wants to reach, Through progressive training, teenagers to boxing to have certain understanding, can effectively increase the reaction ability, mental state, power quality, coordination and a series of physical quality.

Teenagers are in the process of claim gold stage, through the training, to be able to bring in the growth of bone certain external benign stimulation, promote the balance of organic matter and inorganic matter in proportion, bones promote skeletal maturity. Through research, people have regular practice of martial arts, which can improve the body's function and the quality of the bones. In martial arts at the same time, stressed body coordination achievement method in practice, for the deep muscles and muscles, make muscle constantly overcome resistance. This routine practice improves muscle strength, so as to ensure the activity of learning and life. Many of the habits in life are formed in children's time, just like fitness habits.

The Positive Guiding Effect of Chinese Traditional Martial Arts on Middle Age. Middle age is the mainstay of the motherland, is the important force of the construction of the motherland, the city too fast pace of life can also give people certain pressure and mood. Stress of the middle-aged group, in terms of fitness and experience are lost the most exuberant rhythms, physical and mental recovery time is longer, as the physical and mental function decline and many contradictions and confusion in real life, often affect sleep, health and mood. According to the research, middle-aged people are gradually entering the ranks of chronic diseases and mental illness, so it is urgent for the middle-aged people in China to pay attention to their health. In middle age people body various aspects quality has grown up, but too big to exercise load sport has heart tired, and need to be in the cultivation of the exercise habit and self-identity to relieve brings the huge pressure of work and society.

Bthis Chi is a Chinese traditional martial arts sport which is very suitable for middle-aged people. "Tai chi peace the world, Bthis Chi unified the universe" is the words of emperor Qianlong to The Bthis Chi. The " Bthis Chi" means that it can reach far and wide in all directions. Its action is simple and simple, just strong and crisp, the more shake feet vigorous action. The Bthis Chi fists are mainly rigid, like mount tai, moving like a bow, like thunder, lightning fast. The Bthis Chi punch is characterized by its strong, fast and sudden style of boxing, and it is attracted by martial arts lovers who are close to the fight. Practicing Bthis Chi to relieve the pressure of middle-aged people's working life and increase their concern about their own health; In the long run, the Qigong training of Bthis Chi can delay the decline of physiological function and have a benign effect on the function of the body. Reduce your body fat percentage, adjust your body's metabolism, and resist the health effects of cardiovascular disease.

The Auxiliary Improvement of Chinese Traditional Martial Arts in the Physical Condition of Middle-aged and Elderly People. Tai chi exercise groups to practice more for the elderly, the group has a respiratory function for this stage begins to fade, the salient feature of insufficient strength, vitality, Tai chi can increases the strength of respiratory muscles, the form of thoracic mobility and breathing, by practicing Tai chi can increase the pulmonary ventilation, ventilation function wait to have a good effect, for the prevention and treatment of senile chronic bronchitis, 
asthma and other diseases have a certain role.

Fluent speaking and written the study found that after training, the elderly appetite increased by $15 \%$, memory, appetite decreased by $7.5 \%$, while generally reflect the phenomenon such as anorexia, bloating, constipation decreased significantly, but there was no significant difference before and after training. It shows that Tai chi can improve the digestive function of old people. With the increase of age, the decline of the muscle strength of the elderly, especially the weakening of the muscle group, has caused a lot of inconvenience to the elderly. With literature reports, the 65 - year - old muscle strength was worth only $50 \%$ of 20 people, due to the loss of muscle strength degradation of body movement, has been slow to act, so prone to fall down to the life of old people.

Through Tai chi, it can effectively enhance the muscle strength of the old people's lower limbs, the shape of the spine and the prevention of muscle atrophy, and the positive role of bone hyperplasia to prevent the elderly from falling. Illinois university published a research report pointed out that the "tai chi" and "Qigong" way of practice, combining with the physical treatments, such as can delay the symptoms of Alzheimer's disease, and take special drugs to control the condition of the result is closer.

\section{The Value of Chinese Traditional Martial Arts to Mental Health.}

The value of martial arts to mental health is mainly manifested in the conviction of self-identity, self-positioning and self-knowledge and the knowledge of martial arts. Film and television as a media has had a huge propaganda force on martial arts, more and more people feel martial arts, chase through film and television works, when Bruce lee in Kungfu movie becomes the Kungfu has become a way people pursuit vogue. Li Lianjie, a Chinese traditional martial arts athlete, made his mark on the big screen in Hollywood, the world's film factory. They are regarded as the idols in their hearts, depicting the dream images that they have become famous through their martial arts. Participate in Chinese traditional martial arts to the rising number of people, an important reason is the warrior spirit by amateur pursuit, and can change your life, and the effects of positive mood change, as well as the shaping of personality, and even some people have a long stay time, widely participate in friendly games, activities, and to expand your social circle, increase the self-cognition and sense of worth.

Many folk martial artists have experienced a lifetime of martial arts, still feel that they did not achieve the desired state. What is missing in many sports today is the attraction power of this artificial life, an implicit record pursuit. Different from the western "higher and faster" sports spirit, it is more a psychological pursuit. Martial arts have been rooted in people's life, his craft process is the body the feeling of technology process, perception of self, and in the process of the body to body can fully feel the skill enhancement changes, which connect to the implicit body change is essentially the traditional martial arts of the "record". The process of daily practice is to surpass the past self, yesterday is today's contrast, each transcendence is a new impetus to the tireless practitioners. The martial arts people have already developed a bidirectional dependence on this activity, which not only serves as a tool for strengthening physical fitness, but also determines their psychological positioning and self-worth through the practice of martial arts.

The cultivation of martial morals is the cultivation of the healthy personality of human beings. Martial morals need to hold on for a long time, in the inner circle facing his own delicate grasp and control. The first spirit of every martial art in history is bravery and sacrifice. Ordinary people have no chance in battlefield, but life habits and self-shortcoming is our "enemies", hinder our progress, through training to improve their willpower to control, to abuse themselves. The tradition of "respecting the teacher, being upright, being humble and loving" can improve the self-cultivation of the people and build a harmonious interpersonal relationship. The sense of fair competition and good competitive mentality are strengthened in martial morals. The impetuousness of the social atmosphere makes people quick and quick, and the spirit of "self-improvement, the virtue of virtue" and the will quality of overcoming difficulties provide more motivation to move forward. The cultivation of martial morals is to make people make the right choice in the face of adversity, and not to be negative about life's ups and downs. 


\section{Conclusion}

People of different ages have different physical conditions. Through the analysis, young children are suitable to practice the martial arts program to improve the physical quality and ensure the study and life of the active reserve. The middle-aged and young people are suitable for practicing simple and simple, fresh and strong Bthis Chi, delaying the declining physiological state and resisting the healthy erosion of cardiovascular diseases. And because the body function decline, the elderly often appears wrestling cause fracture and even the phenomenon of death, so slow motion meat Tai chi is very suitable for old people practicing Chinese traditional martial arts sport, enhance the function of the respiratory system against respiratory diseases, strengthen the power of the lower limbs fall prevention, effectively prevent dementia. The psychological effect is to be confident in self-identification, self-positioning and self-knowledge and knowledge of martial arts.

\section{References (In Chinese)}

[1] Z.Y. Chen and K.Y. Tao: Sports Science, (2012) No.32(09), p.52.

[2] J. Chen: The research on the national spirit identity of college students in traditional martial in Lanzhou (Ph.D., Northwest Normal University, China 2016), p.9.

[3] C.L. Tang: The first "national fitness, science movement" academic exchange conference proceedings (Jiangxi, China, July 22-23, 2016)

[4]C.L. Tang: On the promoting effect of traditional martial arts on national fitness (Jiangxi, China, July 22-23, 2016)

[5] Z.J. Zhang, Y.X. Long and B.G. Zhang: China Sports Technology, (2016) No.52(02), p.3.

[6] H.B. Yan: The research of martial arts films to promote the cognition of martial arts concept (Ph.D., Suzhou University, China 2015), p.44.

[7] H.D. Liang: Carry forward the martial arts culture and promote the person's all-round development (Taipei, China, April 6-8, 2014)

[8] H.D. Liang: Proceedings of the second cross-strait academic symposium on sports history (Taipei, China, April 6-8, 2014)

[9] P.X. Qiu: History of Chinese Martial Arts (Beijing: Advanced Education Press, China 2008), p.9.

[10] J.B. Yuan: Research on the social influence of martial arts in contemporary China (Ph.D., Fujian Normal University, China 2011), p.17.

[11] Y.Z. Li and H. Yang: Contemporary Sports Technology, (2016) No.6(24), p.10.

[12] W.N. Zheng: Journal of Physical Education of Shanxi Normal University, (2006) No.04, p.49. 